\title{
Chemiosemiosis and Complex Patterned Signals: A Chemosemiotic Hypothesis of Language Evolution
}

\begin{abstract}
Olfaction, as a semiotic modality, receives relatively less attention than other sensory modalities. However, chemiosemiosis and semiochemicals are fundamental components of zoosemiosis, occurring across animal taxonomic groups. Indeed, olfaction is thought to be one of the most ancient sensory modalities from an evolutionary perspective and significantly, even unicellular organisms, such as the bacterium Escherichia coli, utilize a form of chemiosemiosis when foraging for nutrients, as part of a process known as 'chemotaxis'. Further, many taxonomic groups have evolved to produce dedicated 'semiochemicals' (often known as pheromones or allomones) which have the sole purpose of being diffused into the environment as a social signal. In this paper, I highlight the importance of Umwelt theory when studying animal communication, by reviewing the less conspicuous and intuitive chemiosemiotic modality, across animal taxa. I then go on to discuss chemiosemiosis within a linguistic framework and argue that complex pattern recognition underpins linguistic theory. Thus, I explore the concept that chemiosemiosis has features in common with language, when the factor of time, in the transmission and decoding of a signal, is taken into account. Moreover, I provide discursive evidence in support of a unified theory of sensory perception, based on structural and functional aspects of signal transmission and cognitive complex pattern recognition. I conclude by proposing a chemosemiotic hypothesis of language evolution.
\end{abstract}

Keywords: Olfaction; chemiosemiosis; sensory perception; language evolution; animal behaviour; animal communication; cognition

\section{INTRODUCTION}

Chemiosemiosis receives relatively little attention compared to other sensory modalities, perhaps because chemical signalling is complex in nature, and detecting and isolating specific olfactory signs and signals is difficult. Furthermore, it is the visual and auditory channels on which humans primarily rely in our anthropic observations of animal communication and social behaviour, and thus, it is these which seem to receive the most attention. Nonetheless, in this article, I aim to demonstrate just how important chemiosemiosis is to the animal kingdom, and how it has driven evolutionary pathways in animal communication; hypothetically, even language evolution.

Chemoreception involves the detection and perception of chemical compounds by an organism. Generally speaking, the sensory channels involved include olfaction (the sense of smell) or taste (the sense of flavour) (Peng et al. 2015), the latter being a gustatory phenomenon.
Olfaction, however, specifically involves the perception of chemical signs which are transmitted, either from objects in the environment or living organisms, as odorants or scents. Odours/scents are volatile molecules which can be detected and processed via an animal's olfactory sensory channel (Mollo et al. 2014), and it is the olfactory sense, in the context of chemical signs and signals or 'chemiosemiosis', which will be the focus of this paper. Further, whilst the term 'chemiosemiosis' also describes chemical signalling at a cellular level, this article focusses on whole organism signalling, particularly social signalling between individuals of the same or different species.

In biological sign systems, signs represent objects in the environment, with the sign then being perceived by the interpreter (Queiroz 2011) and this process occurs over time. It is a key point that without time as a factor, 
no sign processes can occur. Further, time also mediates the transmission of the signal with respect to the speed of delivery of its component parts and the speed with which a sign or signal is detected and analysed after transmission. Thus, sign processes allow for signs or social signals to be transmitted either all at once or sequentially, and one prominent form of sequential information transfer is human language. Language is a highly complex form of signalling; however, humans are not the only organisms to use complex communication, and there is a growing body of work which focusses on complex vocal signalling in non-human species. Moreover, the use of non-verbal language has been employed to train ape species which are capable of basic non-verbal language acquisition (Call 2011).

The concept of language involves sequencing linguistic constructs and symbols to relay codes or information which, as a cognitive process, is a form of complex pattern recognition. Significantly, however, studies investigating animal communication, such as Berwick (2011) and Favaro (2020), tend to focus on sequential patterns, yet olfaction demonstrates that not all communicative patterned signals are transmitted sequentially. Semiochemicals, which are known as pheromones in intraspecific communication and allomones in interspecific communication, are olfactory signs which are used by some animal species for social signalling and communication. Semiochemicals are evolutionarily ancient (Keller-Costa et al. 2015), pre-dating the other sensory modalities of communication, and olfactory signalling is a primary means of semiosis in many species (Lisberg, Snowdon 2009). Further, these signalling chemicals can be subdivided according to function, for example, on a broad level they are 'primers' (affecting endocrine and neuroendocrine responses), 'modulators' (associated with affective states, such as 'fear'), 'releasers' (which initiate behavioural sequences in the receiver), and the very similar 'signallers' (which transmit information but without a behavioural response in the receiver). For a more detailed account of human pheromones and the functions of these four groups (with some information about pheromones in other species), the article by Wysocki and Preti (2004), is recommended, as it gives a comprehensive overview of the topic. Moreover, pheromones can be further subdivided according to their specific functions, including (but not limited to) 'aggregative' (Siljander et al. 2008) or 'aggressive' (Wang, Anderson 2010); thus, pheromones are a complex means of signalling, and organisms have evolved not only the capacity to perceive meaning, but also the means to produce the chemical compounds, which have meaning attributed to them by the receiver.

Moreover, a prominent feature of semiochemical signalling is that components of the signal are transmitted simultaneously, rather than as part of a linear sequence. Thus, neurologically, semiochemicals form complex nonlinear patterns for the interpreter (Baird 1986). Indeed, machine learning and pattern recognition are now techniques being employed to study different aspects of olfaction and chemiosemiosis, including the physio-chemical properties of odour molecules and the physiological response of the interpreter (Lötsch et al. 2019).

Given the complexity of chemical signalling within, as well as between, taxonomic groups, I thus explore the possibility that complex messages can be transmitted non-sequentially, and moreover, that human language is, in fact, a non-linear pattern which could have its origins in chemiosemosis, which appeared very early in the tree of life. I therefore highlight the importance of Umwelt theory when studying ethology in order to avoid focussing research paradigms and data interpretation through an anthropocentric lens. To do this, it is necessary to review the olfactory capabilities across taxa in different species and taxonomic groups. By doing so, the rich diversity of chemical signalling-along with common underlying factors-becomes apparent and reveals that chemiosemiosis is a key component of zoosemiosis. Not only that, but as a method of sensory perception, it is intricately interwoven with the other senses. Thus, I reviewed the literature to find examples of some of the diverse chemiosemiotic systems in the animal kingdom, starting with our own taxonomic group: Mammalia.

\section{MAMMALIA}

Mammalian olfaction initially takes place in the nasal apparatus, across an olfactory epithelium (Reed, 2004). Many mammal species, including (but not limited to) ungulates, rodents, felids, lagomorphs, and canids, have a vomeronasal organ (VNO). Located at the anterior of the nasal cavity, the VNO collects chemical compounds which then act as olfactory signs, often in relation to social signals from conspecifics. Whilst a feature of the mammalian anatomy, the VNO is also found in amphibians, from which taxonomic group it is thought to have originated (Villamayor et al. 2018; Døving, Trotier 1998). Semiotically, the VNO is key in social signalling and social interaction, including sexual reproduction; the VNO detects olfactory signs which are key in mediating courtship and mating (Curtis et al. 2001) as well as parental care (Villamayor et al. 2018). Indeed, one olfactory behavioural response familiar to horse owners (and which occurs in other ungulate species) is the Flehmen Response (figure 1), where the horse, Equus caballus, retracts the upper lip to bare the teeth and compresses the tissue of the nose, giving it a wrinkled appearance, often with the neck extended (Weeks et al. 2002). Whilst previously linked to olfactory signs related to socio-sexual behaviour, the Flehmen response in horses has also been linked to various other phenomena, including mediating maternal care, and in the analysis of novel olfactory signs. Indeed, Saslow (2002) and Weeks et al. (2020) conclude that the specific socio-sexual function of the organ in horses is still not fully understood, but olfaction is key in the horse's social behaviour (figure 2). Significantly, the sensitivity of the olfactory sense in horses is such that 
horses can discriminate between the body odours of conspecifics, and it has even been suggested that horses can recognise human emotions from olfactory signs (Sabiniewicz et al. 2020).

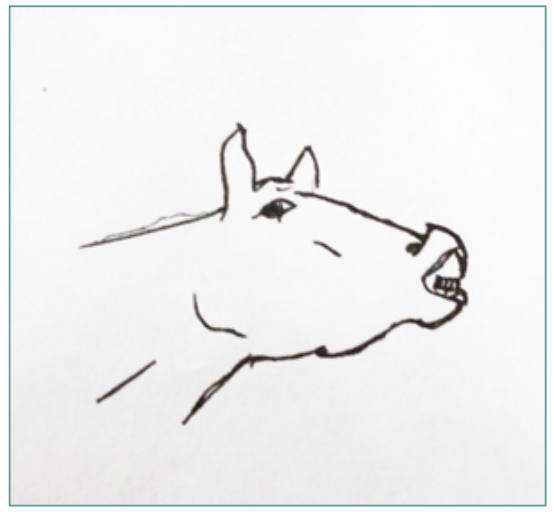

Figure 1: Diagram showing Flehmen response in a horse. The upper lip (and to some extent lower lip) is retracted, and the neck and head are extended. The oral mucosa is exposed, and the nose has a wrinkled appearance.

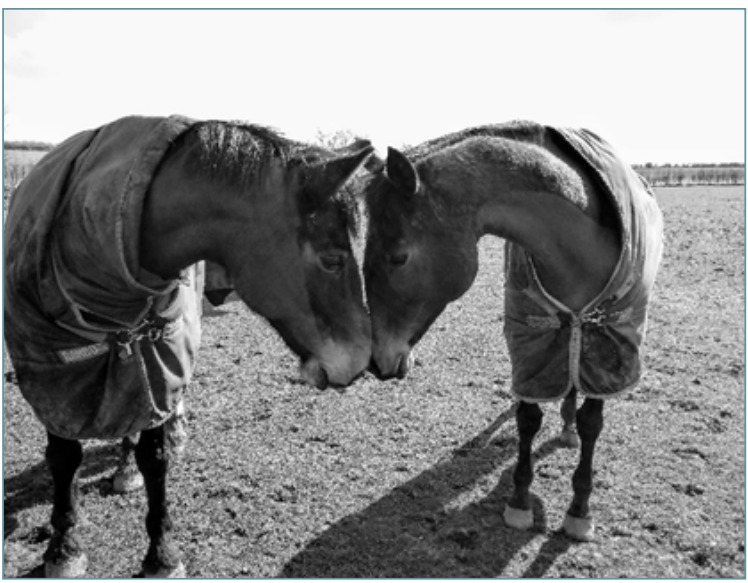

Figure 2: Social behaviour is facilitated by

chemiosemiosis in the domestic horse, Equus caballus.

Another mammalian species with a highly developed olfactory sense is the dog, Canis lupus familliaris, a descendant of the wolf, Canis lupus. So advanced are the dog's olfactory capabilities, that humans have harnessed them to their advantage. Domestic dogs are used to aid in search and rescue, drug detection, police tracking of suspects, cadaver detection (Jones et al. 2004), and most impressively, disease detection and diagnostics (Wells 2012), including detection of the SARS CoV-2 virus during the 2020 COVID-19 pandemic (Jendry et al. 2020). Indeed, unlike humans, olfaction is a primary modality by which dogs sense the world, as illustrated in figure 3 .

Having such a highly developed olfactory sense means that olfactory sign processing allows for complex social signalling. Indeed, the domestic dog can transmit information to conspecifics relating to individual identity, including sex and social status. One method which dogs use to diffuse chemical signals is urine marking
(Catala et al. 2019; McGuire Bemis 2017; Horowitz 2017; Cafazzo et al. 2012) as illustrated in figure 4. In female dogs, urine marking usually occurs around oestrus, indicating it is a signal related to mate attraction and sexual reproduction. However, urine marking in males, and also, to a lesser extent, females, is thought to be related to territory defence and dominance hierarchies (Cafazzo et al. 2012)

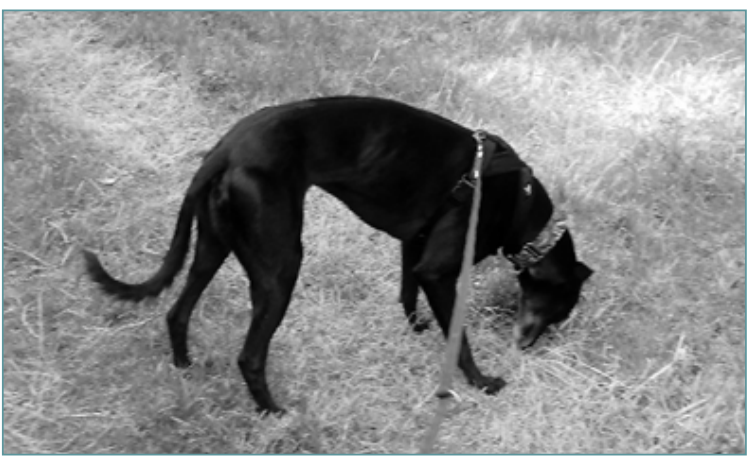

Figure 3: A female greyhound investigating a chemical sign, likely the urine scent-mark signal of another dog.

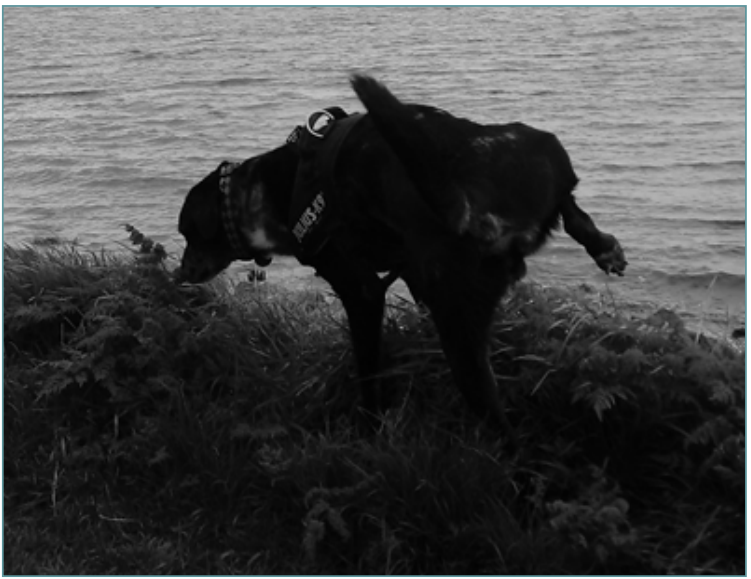

Figure 4: An un-neutered male mixed-breed dog leaving a chemosemiotic urine mark on a patch of vegetation to signal to conspecifics.

To illustrate using a personal anecdote; I lived for many years alongside an un-neutered male mixed-breed dog I had named Sebastian. He had developed a social bond with a male West Highland terrier, Bertie, who lived close to my family's home. Bertie walked by the house with his owner every day, and if Sebastian was in residence, Bertie (not his owner!) would often run up the drive and call at the house. However, if we were away, Bertie would apparently walk by, paying little attention. On one occasion, Sebastian and I had returned to the family home for a visit, from our own house, arriving late in the evening. Sebastian disembarked from the car and promptly went to the front gate, where he urinated on the gate post. Being next to a popular dog-walking route in a rural area, the front gate post is a common spot for dogs to leave their personal olfactory signatures, and so the mix of olfactory signals was likely complex. 
The next morning, however, we were surprised to hear barking and going to the front door, I found Bertie on the porch, waiting to greet his prodigal friend. Shortly thereafter, Bertie's owner arrived, explaining that Bertie had suddenly become excited and run ahead down the lane. Sebastian had not made any vocalisations, nor had any of his excursions into the garden, in the brief period we had been at the house, been visible from the lane or from Bertie's home. It would appear, therefore, that Sebastian's calling card had worked; information relating to numerous physiological characteristics had come together to produce his own unique odour, which had diffused through the environment and alerted his associate to his return, inviting (in this instance) positive social interaction. What is remarkable is Bertie's isolation and detection of an individual scent from a complex chemoscape of odours, which make up part of his and Sebastian's Umwelt.

It is curious that whilst I referred to my dog as Sebastian, I actually had very little conscious knowledge of his real 'name', which was coded in various chemical signs. In my own species, a human will sign their title and names sequentially, according to cultural traditions and etiquette. For example, in the UK, the traditional sequence would be an honorific, which traditionally indicates sex or gender (Mr, Master, Mrs, Miss, Ms), and (for women) marital status (Mrs/Miss), age (Mister/ Master) social rank (Lord/Lady, Rt Hon, Prince/Princess, Sir/ Madam, etc.), and in some instances, profession (Doctor/Dr, Professor/Prof, Reverend/Rev), then a forename, middle name(s), surname or family name (which denotes paternal ancestry), followed by postnominals, which indicate someone's level of education and professional memberships. Sebastian's personal data, however, was transmitted simultaneously, and the signal contained sufficient information for him to be identified from the numerous other urinary signals left by the many dogs (and possibly the local fox population) who passed by the house. Nonetheless, from a human perspective, Sebastian's data was transmitted all at once with no sequence involved-or certainly, no sequence that has yet been identified.

Another relevant example in dogs (as well as other carnivores, including various canid species and bears) is their tendency to roll in scent; for example, the excrement of other species, or dead and decaying organic matter (Reiger 1979). Traditional ethology has struggled to reach a consensus on the function of this behaviour, putting forward various theories, the most common being that scent-rolling is a form of camouflage to avoid larger predators or mask an individual's own scent from prey (Gray 2017). Semiotically, this signalling therefore appears to be a form of either mimicry or masking, a detailed description and account of the different forms of mimicry being given by Maran (2017). It would, however, be useful to investigate the odour compounds common to the substances dogs commonly roll in, to attempt to isolate the signal being transmitted, before trying to deduce meaning from the behaviour.

\section{HUMAN CHEMIOSEMIOTIC CAPABILITIES}

Unlike dogs, humans do not overtly rely on the sense of smell as a primary communicative sense. Despite this, however, humans have a very sensitive and advanced olfactory system, and a comprehensive review by Zelano and Sobel (2005) provides a detailed description of the relevant anatomy and physiology. Despite not being a primary sensory modality, olfaction has a variety of important semiotic functions in humans, including during gustatory behaviour, and in recognising and avoiding potentially harmful substances and environmental hazards. Physiologically, humans also produce chemicals which are involved in mediating social and reproductive behaviours (Stevenson 2010); thus, chemiosemiosis is an important cornerstone of human behaviour and culture. Indeed, one fascinating aspect of human culture is the use of 'perfumes'. Many cultures and ethnicities use perfumes as social signals, either by applying a scent to the body or clothing, or by diffusing them in the environment as aerosols, or by various means of evaporating volatile chemical compounds (Panda 2010). An example of a 'Bakhoor burner' can be seen in figure 5 .

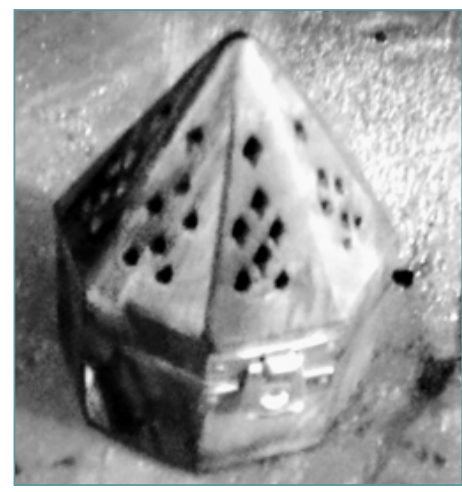

Figure 5: A smoking 'Bakhoor burner', popular in Middle Eastern cultures. Wood chips (Bakhoor) soaked in aromatic plant-based perfume oils are burned over smouldering charcoal. The scented smoke diffuses into the atmosphere to perfume rooms, clothing, and people's hair.

These practices can be traced back thousands of years, yet from an evolutionary perspective, the significance is not fully understood. However, what is known is that perfumes form a complex interplay between natural body or environmental odours and the perfume (Havlíček Roberts 2013) with an effect that humans find pleasant. Nonetheless, semiotically, perfumes are either a form of mimicry, or mask unpleasant body odours and environmental odour. The signal produced by a perfume is complex, and a 'perfumer' mixes fragrance in a solvent to create a scent, which can be diffused into the environment to be perceived by others. The art of the perfumer is to produce a mixture which will be appealing, and to achieve this, a mixture of scents is created in solution to form a harmonious blend. Thus, a complex molecular signal is released, interpreted and can be described 
linguistically as 'notes' of floral, musk, citrus, fruit, woody, spiced, or whatever perceived meaning the scent evokes. Strikingly, the effect is similar to acoustic harmonics in a piece of music, and as such this is another example of complex sensory pattern recognition.

Semiotically, the significant point in relation to perfume use and the 'scent rolling' behaviour in canids is the decoupling of the olfactory sense from autonomic nervous system control and physiological processes. Species capable of scent use are able to use odours in the environment, and this gives them conscious control over a sign. Further, odorants can be adapted, altered, or given context, to give them iconic meaning; for example, gender-specific perfumes and fragrances, and pine and citrus scents being associated with cleanliness. This means there can be a degree of abstraction to the signal produced, and significantly, this is a phenomenon found in language.

\section{AVES}

Birds are well known, as a taxon, for being vocal, particularly the songbirds which produce elaborate and complex vocal sequences which have been compared to human language (Berwick et al. 2011) and are musical to the human ear. However, olfaction is not a form of sensory perception readily associated with birds, but despite this, current research indicates it is a highly developed sense in avian taxa. One of the most intriguing discoveries in more recent research is that juvenile zebra finches can identify their natal nest using odour signs (Caspers, Krause 2011) and furthermore, the use of aromatic plants to line nests by blue tits is now thought to be a sexually selected trait in females, which serves to attract male mates (Tomás et al. 2013). Moreover, male starlings have also been found to use olfactory signals to select aromatic herbs to line nests (GwinnerBerger 2008), and indeed, colonies of blue tits surviving in habitats where aromatic herbs are available line their nests with herbs, whilst in environments where no aromatic herbs are available, alternative non-aromatic plant species are not used (Lambrechts Dos Santos 2000). Thus, this indicates a distinct preference for aromatic compounds, such as lavender, pictured in figure 6 .

The hypothesis to explain this curious behaviour is that the function of aromatic herbs in nests is generally accepted to be anti-pathogenic and anti-parasitic, thus keeping the natal environment healthy for juveniles. The common factor across these examples is that they all involve odour signs, and in the case of species using aromatic herbs as a sexually selected sign, the odour has become an iconic signal of 'attractiveness' to a potential mate; thus, a striking parallel can be drawn with perfume use in humans.

Further examples of social signalling in birds can be found in a paper by Bonadonna and Sanz-Aguilar (2012) who give an overview of studies which have found olfactory kin recognition to be present in various bird

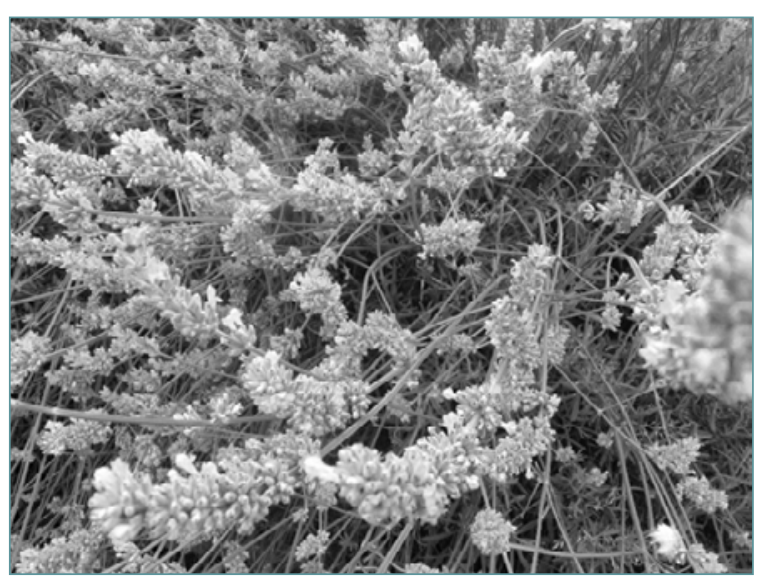

Figure 6: One of the several species of Lavender (Lavendula), an aromatic herb known for its anti-bacterial properties. Used both in human aromatherapy and perfumery, and by some songbird species to line their nests.

species, including zebra finches. The authors then go on to present their own results from experiments on kin-recognition in European storm petrels. Taxonomically, petrels are Procellariiformes, an order of seabirds which includes four families. The fascinating life histories of these ocean-going birds involves annual group nesting in breeding colonies on remote islands off the coast of Europe, where there are no natural ground predators to disturb nesting pairs, or their juvenile nestlings. The lack of natural predators makes these safe breeding grounds a limited resource, resulting in populations forming large breeding colonies with numbers totalling hundreds of thousands of individuals. Furthermore, petrels are philopatric, meaning that all fledglings return to their natal breeding site to breed as sexually mature adults, on an annual basis. This gives rise to the risk of inbreeding in a population, and so kin recognition is a vital aspect of social behaviour to ensure mated pairs are not genetically related. In petrels, this recognition of relatives is achieved via chemiosemiosis. Indeed, the impressive olfactory sense in petrels is one of the most highly developed of all seabirds, giving rise to complex chemiosemiotic capabilities. Mechanistically, petrels secrete chemicals in the form of waxy secretions from the uropygial (preen) gland, which contain individual chemical signatures (Bonadonna Sanx-Aguilar 2012 citing Bonadonna et al. 2007). It is this unique chemical signature which allows for individual recognition, based upon memories of specific chemical signatures of kin. Thus, this avoids the substantial risk of inbreeding in large philopatric colonies where predation risks from ground predators is non-existent, giving colony members a reproductive advantage.

\section{POTENTIAL LINKS BETWEEN \\ CHEMIOSEMIOSIS AND GUT PHYSIOLOGY}

Another interesting avenue to explore in avian taxa is the odour produced by body microbiota, as this may be 
important in individual recognition and other aspects of avian social behaviour. Maraci et al. (2018) discuss body odours arising from avian body microbiota, including microbes found on the skin, uropygial gland, and feathers. Further, the authors describe the effects of gut microbiota on the odour of faeces, and conclude that in domestic poultry, faecal odour can be an indicator of gut health, with less odorous droppings indicating an individual with a healthy enteric gut microbiota. Certainly, in the case of gut microbiota, any olfactory signs from the enteric microbiome would be complex in nature, given the diversity of microorganisms in the gut, which includes bacteria, fungi, protozoa and viruses (Maraci et al. 2018).

One factor all the previous avian examples have in common is that these are complex olfactory patterns involving more than one compound, which are transmitted simultaneously to be interpreted as olfactory signs or signals. Further, in the examples of blue tits and starlings, we once again observe the behavioural use of environmental scents; an elective behavioural phenomenon which has been decoupled from those bodily physiological processes which produce odour.

\section{REPTILIA AND AMPHIBIA}

Most amphibian and reptile species (except crocodilians) have a vomeronasal organ (VNO) which, as in mammals, facilitates olfactory sign processes in relation to predation, predator avoidance, and recognition of conspecifics (MillerGutzke 1999). For example, rattlesnakes (Crotalinae) are a group of pit vipers of the family Viperidae which 'rattle' their tail as a predator avoidance mechanism. The rattle is employed when the snake encounters large predators, but interestingly, not when threatened by ophiophagous snakes. The explanation can be found in the fact that rattlesnakes are predated upon by kingsnakes, Lampropeltis getula, and a study by Miller and Gutzke (1999) found that rattlesnakes are able to detect kingsnakes from a semiochemical produced in the skin of the kingsnake. It is this signal which prevents initiation of the tail rattling behaviour in the rattlesnake, whilst simultaneously initiating more successful and thus appropriate alternative predator-avoidance and defence behaviours, including body inflation, body bridging, or retreat. Indeed, Schwenke's (1995) review concludes that lipid secretions by the integument are one of the primary sources of semiochemicals in squamates. Thus, rattlesnakes receive an olfactory signal which not only alerts of an approaching predator, but also gives information on the type of predator, allowing them to mediate their response accordingly.

Schwenke (1995) also goes on to discuss the role of tongue flicking behaviour in snake and lizard species, and the relation of the behaviour to the function-the VNO. Tongue flicking detects non-volatile chemicals, which take longer to diffuse through the environment and thus are 'captured' by the tongue and transferred to the VNO. Therefore, in relation to olfactory signs in an environment's chemoscape, complex patterns are formed which rely on the timing of signal reception and composition of an odour, in order for them to be identified by the interpreter.

Amongst further studies on amphibian taxa, a study by Zeng et al. (2016) revealed that anuran olfactory bulbs are larger in frog species which are more vocal during courtship and maintain monandrous mating systems. From their data, the authors conclude that this is evidence for the neo-Darwinian social brain and mosaic evolutionary hypotheses. However, an alternative interpretation employing a structuralist approach would suggest that social behaviour, sensory perception, and communication are intrinsically linked on a semiotic level, meaning that it is sensory perception and energetic physical phenomena which are driving evolutionary processes, as suggested in my own paper (Lewis 2020) which puts forward a hypothesis for a unified theory of sensory perception.

\section{CROSS-MODAL SENSORY PERCEPTION IN REPTILES}

Despite being the only reptile group without a VNO, olfaction is still an important sensory modality for crocodiles. Indeed, a study by Chabrolles et al. (2017) revealed that Nile crocodiles Crocodylus niloticus rely on both olfaction and vision during appetitive gustatory behaviour and employ cross-sensory modulation when selecting food sources. This means that the visual sign must match the olfactory sign for a food source to be considered edible and consummatory behaviour to be stimulated.

Cross-modal integration has been found in numerous species, including goats (Pitcher et al. 2017), dogs (Albuquerque et al. 2016), and horses (Proops et al. 2009) and sensory integration occurs throughout the brain. However, one brain region where the activity is particularly pronounced is the superior colliculus (Meredith Stein 1986) and furthermore, a study on cats found that monomodal neurons in the superior colliculus were, in fact, able to receive and process subthreshold stimuli from more than one sensory modality, which may influence sensory processing of cross-modal sensory input (Allman and Meredith, 2007). Thus, further support for the idea of a unified theory of sensory perception exists. Moreover, this evidence indicates highly complex integrated pattern recognition capabilities in many animal species.

\section{FISHES}

\section{OSTEICHTHYES}

Amongst teleost species, one of the taxonomic groups yielding a significant amount of data on semiochemicals are the cichlids. A diverse and widespread group, cichlid species have been found to form breeding pairs, they engage in parental care, and their social groups show complex social behaviours, including the formation of dominance hierarchies and territorial defence. Indeed, 
socially affiliative behaviours in one cichlid species, $\mathrm{Ne}$ olamprologus pulcher, have been found to provide a social buffer with the presence of familiar conspecifics reducing corticosteroid levels after a stress response (Culbert et al. 2019).

Furthermore, chemiosemiosis is an important part of cichlid reproductive behaviour. Cichlid mate choice experiments have revealed that reproductive isolation and allopatric speciation in Lake Malawi cichlids is primarily driven by female preference for conspecific males based on chemosemiotic signals from males (Plenderleith et al. 2005). Moreover, reproductive olfactory semiosis goes beyond mate choice and is found to mediate parental behaviours in species showing parental care. For example, in mouth brooding cichlids, such as Astatotilapia burtoni, electro-olfactograph studies have shown that the olfactory response to amino acid chemical signals mediates both reproductive and feeding behaviour, which is vital to prevent the fry in the female's buccal cavity from being eaten by the female during mouth brooding (Nikonov et al. 2017). This example indicates a complex semiotic interplay between sign processes originating from physiological, behavioural, and environmental states.

What can thus be demonstrated by the cichlid group is that complex social behaviours require social signalling to co-ordinate and maintain them, and to allow communication to take place. Indeed, significantly, cave-dwelling males of the species Pelvicachromis taeniatus are able to recognize their own semiochemical signature, and thus its own cave and territory (Thünken et al. 2009). This gives scope for semiotic study into animal self-recognition, where visual tests such as the mirror test have previously been unsuccessful (Horrowitz 2017) because they have failed to take into consideration the Umwelt of the species tested.

However, chemiosemiosis occurs in other teleost species, for example, a very familiar domesticated teleost which uses semiochemicals is the goldfish, Carassius auratus. Sexual reproduction is almost entirely facilitated by semiochemicals, with the production of hormones by the female ('releasers') initiating a series of mating behaviours in the male, which involve pushing the female into aquatic vegetation where the female ovulates (see figure 7). As eggs are released into the water, the male releases sperm in synchrony and the eggs are fertilized, where the resulting fry embryos develop and hatch in the shelter of vegetation (Sorensen et al. 1988).

\section{CHONDRICHTHYES}

Cartilaginous fish also use chemiosemiosis as a primary means of sensory perception, although there is considerable variation in olfactory bulb size and olfactory sensitivity between shark species (Yopak et al. 2015). Hammerhead sharks, of the family Sphyrnidae, are a group of species with an acute olfactory sense, and they have been studied because of their anatomically broad head, large distance between the nares and highly

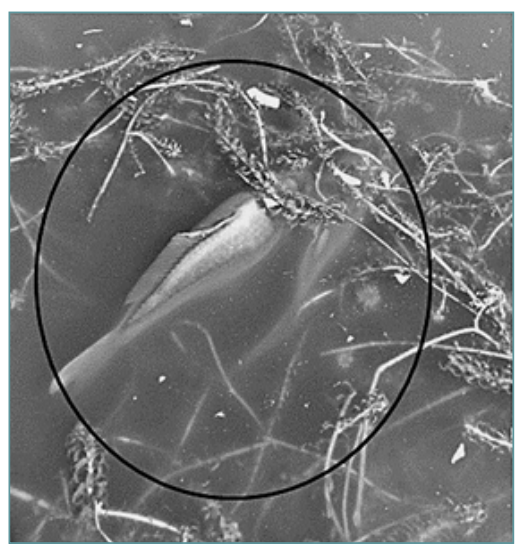

Figure 7: Goldfish breeding behaviour. A female goldfish (right) is shunted into a patch of Elodia by a male (left) where she will spawn. The male will release sperm to fertilize the eggs.

developed olfactory epithelium (Tricas et al. 2009). Indeed, hammerhead sharks have been found to be able to orient to olfactory signs when tracking a chemical plume or odorant-based prey trail through the ocean. Individuals use the time of arrival of odour molecules at each naris to orient their position in the water, thus allowing an individual to track the chaotic diffusion of odour molecules through the water (Gardiner, Atema 2010). Whilst it could be argued that the shark simply responds behaviourally to a stimulus, the fact that this is a process which is repeated sequentially over time in response to a changing concentration of complex odour compounds suggests that not only does it involve neurologically complex pattern recognition of individual odours, but that the whole dynamic process of tracking a complex chemical plume in itself involves pattern recognition i.e., the frequency of odour molecules arriving at, and being detected by each naris.

\section{INVERTEBRATES}

The term 'invertebrate' covers a large and diverse group of phyla, the majority of which (to the best of my knowledge), all have some form of chemosensory abilities. Cladistically, the term includes any taxonomic group which has not developed a vertebral column, and thus, to cover the range of chemosensory abilities across invertebrate taxa would not be possible within this article. Therefore, this is a small selection of some of the extensive examples of chemiosemiosis across an extraordinarily diverse and large number of taxonomic groups.

\section{MOLLUSCA}

Mollusc species rely on olfactory signs which are detected across the epithelium, the chemical sign primarily being metal ligand groups in an odorant. Indeed, Croset et al. (2010) suggest that the origins of olfaction in molluscs (and in all protostomes) are ancient. Gastropod molluscs and most famously, limpets, use chemiosemiosis in 
homing/navigational behaviour. Limpets follow mucous trails to return to the small dips on rocks, where they shelter at low tide after foraging expeditions, the shell forming a seal between the atmosphere and the rock to avoid the individual drying out (figure 6). Moreover, limpets are able to use chemoreceptors in various anatomical locations including the tentacles, which detect the scent of predatory starfish species (Philips 1975). Further, slug and snail species, where the olfactory apparatus is found in the second pair of sensory tentacles (lerusalimsky Balaban 2010), shown in figure 9 a and b, have also been found to employ chemiosemiosis in foraging and feeding, aggregative, and sexual behaviours. Indeed, these gastropods also employ semiochemicals in mucous trails, in order to navigate (Emery 1992; Croll 1983).

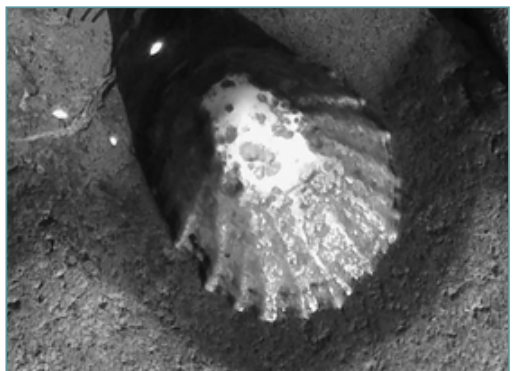

Figure 8: Limpet on a rock in an inter-tidal zone. The limpet returns to the same place after each foraging expedition, at low tide, by following a mucous trail.

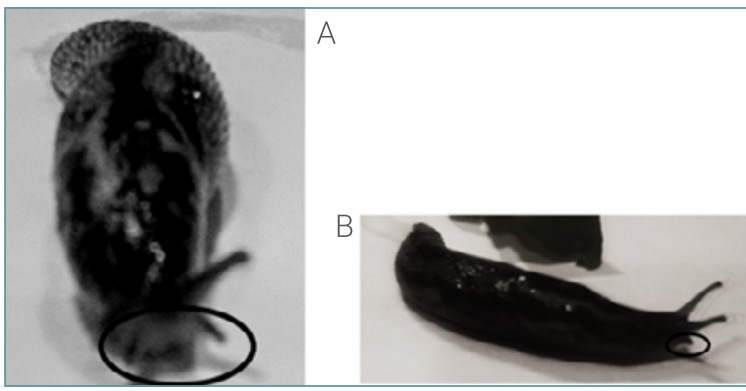

Figure 9: A and B. Leopard slug, Limax maximus. The olfactory tentacles (circled) are positioned on the head below the photosensitive upper tentacles. Both pairs can be retracted into the mantle.

\section{INSECTA}

It is difficult to select examples from such a large taxonomic group with such a diverse range of chemical signalling, but I will start with an example from the Coleoptera. In bark beetle species (Scolytinae), pheromones or semiochemicals are used in sexually reproductive behaviour, with females producing a semiochemical which attracts males and then stimulates a male sexual response. Kaissling $(1996,258)$ reports that "A pheromone blend elicits a particular excitation pattern across the different types of receptor cells which is evaluated by the CNS (Boeckh and Ernst 1987; Homberg et al. 1989). Only the correct pattern of excitations elicited by the species-specific pheromone blend leads to a successful behavior of the male."

Numerous examples of chemiosemiosis and the use of semiochemicals can be found in the order Hymenoptera, particularly social species. For example, famously, many ant species use pheromone trails to guide foraging behaviours, and research has revealed that these trails are complex, with individual species employing several different pheromones when foraging and nest building, including leaving complex chemical trails to recruit and guide conspecific nest mates. Moreover, the complexity of some ant species' pheromone trails is even more impressive; some species use volatile and non-volatile pheromone variants to leave a chemical 'memory'. This group memory functions by worker ants leaving volatile chemical trails leading to low-yielding foraging patches, where those patches are depleted quickly. As the compounds are volatile, the trail evaporates more quickly than non-volatile trails. The non-volatile trails, on the other hand, lead to more lucrative nutrient supplies, thus the chemical sign lasts longer so that more workers arrive at the more abundant nutrient source over time (Jackson Ratnieks 2006).

Lepidopteran species also employ olfactory signs, which are received via olfactory sensory neurons in the legs, proboscis, labial palps, and antennae. Cross-modal sensory perception has been found to be important in some species, for example in some tropical nectar feeding butterflies, such as the large tree nymph /dea leucone (figure 10), both an olfactory and visual colour sign must be present from a flower before an individual will start to feed. Significantly, this is similar to the results of studies on cross-modal recognition in mammals and crocodiles. Indeed, in lepidopterans it is thought that this may be an evolutionary adaptation of flowering plant species, which produce volatile compounds as allomones to attract butterflies to facilitate pollination (Sourakov et al. 2012). Thus, chemiosemiosis as a signalling modality may have also driven evolutionary adaptations in plant species.

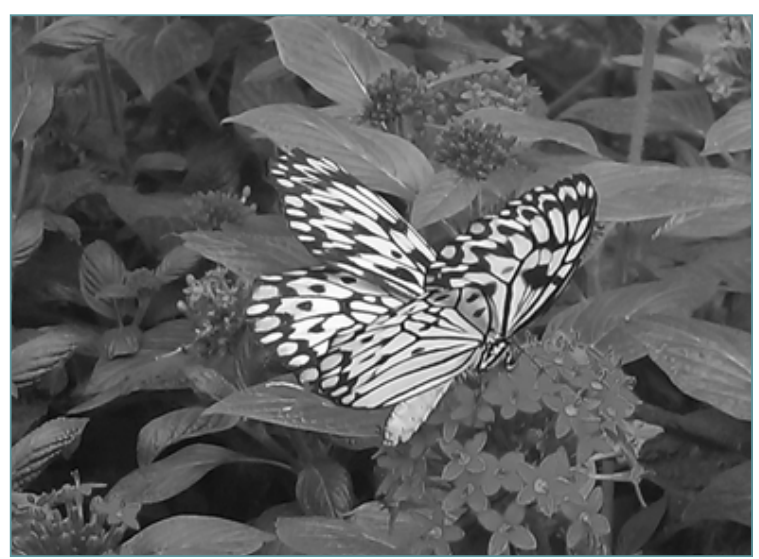

Figure 10: A large tree nymph butterfly feeding from a flower. Sourakov et al. (2012) found that both an olfactory sign and a visual colour sign must be present before feeding commences. 
Therefore, the sensory perceptual abilities of butterflies and moths are not only complex in individual modalities, but these animals are able to integrate complex sensory data from more than one sensory channel.

Strikingly, as in other taxa, the key features in these examples are complex pattern recognition in olfactory cues, as well as evidence of forms of memory and cross-modality in sensory perception.

\section{MICROORGANISMS}

Olfaction is a sensory modality usually associated with multicellular organisms. However, whilst they may not employ olfaction in the strictest sense of the term, bacteria such as Escherichia coli use a form of chemical sensory perception as a method to forage and locate chemically favourable environments, which is termed 'chemotaxis' (Dasgupta et al. 2008). Further, one study has shown that bacteria employ a form of memory to compare concentrations along a chemical gradient, by sampling at time intervals whilst they swim through the substrate. Bacterial cells swim in a series of 'runs', where they move in a straight line, interspersed with 'tumbles', where cells spin, giving rise to a 'random walk' pattern which facilitates a gradient descent search. As the cell moves up the chemical gradient in this fashion, the number of runs can be seen to increase, whilst the number of 'tumbles' decreases (Vladimirov Sourjik 2009). This is because the cell is directed toward high nutrient concentrations in a chemical diffusion. The chemo-sensory process thus mediates the movement of the cell toward abundant nutrient sources or a suitable environment, with relation $\mathrm{pH}$ etc.

In a simple unicellular organism, it might be difficult to make an argument for complex pattern recognition, or what in the strictest sense would be termed chemiosemiosis. Nonetheless, a gradient descent search is guided by a series of simple behavioural responses to an environmental stimulus; this is also similar to the previously described method employed by hammerhead sharks, as they track chemical plumes in the ocean. Moreover, such a process repeated many times over in short spaces of time, along with a capacity for memory, becomes pattern recognition via a process of self-organisation. This ultimately results in emergent phenomenon, as is described by Alexander and Grimes (2017) in their paper about fluid biosemiotic mechanisms. Indeed, it is also the case that varying degrees of complexity can be found amongst bacterial species, with those inhabiting more than one ecological habitat having five times as many chemoreceptor genetic codes than those occupying single habitat niches. Even more astonishing is the fact that species such as Pseudomonas aeruginosa have multiple chemosensory pathways (four, in the case of $P$. aeruginosa (Ortega et al. 2017)), giving rise to a degree of sensory processing complexity even in a microorganism. Thus, examples such as the one found in bacteria and, indeed phenomena such as Lévy walks ( an algorithmic description of the paths followed by foraging multicellular organisms based on Brownian motion (Giuggioli Bartumeus 2010)) could be argued to be iterative stages of higher cognitive functions such as language acquisition, which will be discussed subsequently.

\section{A SUMMARY OF CHEMIOSEMIOSIS ACROSS TAXA}

Whilst this paper is not intended to be a comprehensive systematic review of olfaction and chemiosemiosis across animal taxa, it does provide insight into the complexities involved in olfactory sensory perception and highlights the widespread use of chemiosemiosis in social, physiological, foraging, and reproductive behaviours (with recommendations for further peer-reviewed publications on specific topics being made in the text).

Describing very basic chemical pattern recognition in bacteria, based upon simple binary stimulus-response behaviour, and outlining the gains in sophistication in the higher taxonomic groups, the review also demonstrates the necessity for complex pattern recognition in olfactory/chemical sensory processing. Further, the article illustrates the extent of sensory integration and cross-modal perception in several taxa and describes how it is this sensory integration which adds yet another significant layer of complexity to higher organism sensory processing and pattern recognition. Thus, the foundations for the argument that chemiosemiosis has provided the physiological and cognitive basis for language evolution are laid.

\section{CHEMIOSEMIOSIS AND LANGUAGE EVOLUTION}

As a complex form of social signalling, language is thought to be unique to humans. However, other species have been found to show features of language in vocal communications, including syntax; components similar to phonemes or words (Schmidt et al. 2008), rhythm (Ravignani et al. 2019), and features of some linguistic laws (Favaro et al. 2020). Further, some ape species, whilst lacking the physiological and neurological apparatus to reproduce speech (Ackerman et al. 2014), are able to use lexigrams and sign language to communicate with humans (Call 2011). Whether human or non-human, traditional linguistics studies visually symbolic and auditory signals, so why is chemiosemiosis relevant?

Species of almost every taxonomic group have the ability to detect and respond to chemicals in the environment, and chemoreception is generally accepted to be the most ancient sensory modality (Derby et al. 2016). Whilst humans rely on olfaction as a sensory modality, human communication largely takes place via the acoustic and visual channels; i.e., written and spoken language (Chafe, Tannen 1987) as well as sign language (Clay et al. 2014). Language, however, is only one form of human communication; the visual arts and music are examples of other modalities of human communication, and both 
have more in common with chemiosemiosis, because both involve complex patterns being transmitted and received simultaneously (as well as sequentially, in music). Examples of such complex signals are paintings, most intuitively, abstract and impressionist paintings, and musical harmonies and chords. Nonetheless olfaction, like language, is a form of complex pattern recognition, albeit with the components of the signal being transmitted instantaneously, in a multidimensional complex pattern. Furthermore, chemiosemiosis in some bird and mammal species has become decoupled from physiological processes, and scents have become iconic and to some degree, represent abstract concepts such as 'attractiveness'. Moreover, some fish species show complex chemiosemiotic abilities, including the potential for self-recognition via the olfactory modality, suggesting that latent undiscovered cognitive phenomena may be present in species, because research has not, to date, factored in the differing Umwelt of other animals.

However, whilst some of the examples given in this paper are forms of complex pattern recognition, prima facie, this is not likely the case in the example of E. Coli, which is simply a single cell responding to a stimulus, with no complex cognitive system involved. However, consider what happens when this process is accelerated. A fast series of binary choices (move away OR move toward) in response to olfactory stimuli, i.e., an increased frequency of stimulus-response, ultimately becomes complex pattern recognition when memory is involved Indeed, Jackson and Ratnieks (2006) describe a model in which individual ants are only capable of basic computational decisions according to a set of behavioural rules. However, ant colonies, comprised of thousands of individuals, are capable of complex communication processes and pattern recognition when the individual processes are pooled and the colony acts as a group. The analogy to a neural network in a higher vertebrate is striking, and thus, as unicellular lifeforms evolved into multicellular organisms, the resulting cognitive capabilities may have developed from simple stimulus-response sequences which, when the frequency is increased, become complex pattern recognition. Further, this complexity can give rise to 'meaning' in the minds of higher organisms as an emergent property of groups of single cells. Nonetheless, one of the apparent differences between language and chemiosemiosis is that prima facie, language has a linear sequence as opposed the non-linear patterns of olfactory signals. However, on further examination, how linear is the linear sequence of language?

Firstly, even sequential forms of signal transmission involve patterns. For example, non-adjacent dependencies are one aspect of linguistic pattern recognition which can be tested experimentally in humans and in nonhuman animals (Malassis et al. 2018). Furthermore, the majority of human languages, as well as some animal vocal communications, show prosody (Wilson, Wharton 2006). This involves rhythm (Ravignani et al. 2019), intonation, timing, and amplitude-all varying throughout the sequence-and adds further dimensions to the sequential pattern (Richards Goswami 2019; Wilson Wharton 2006). These features can be observed using acoustic spectral analysis of speech, or in written language, formatting features such as italics, bold, underlining and capital letters. Indeed, even in sign languages, emphasis can be placed on words via the orientation of a gesture in space and time, and additionally, the signaller's facial expression can yield important information in relation to the signal. Thus, the interpreter is not only paying attention to a sequence of words, but also various other associated characteristics of the signal. A pictorial representation of the concept can be seen in figure 11 .

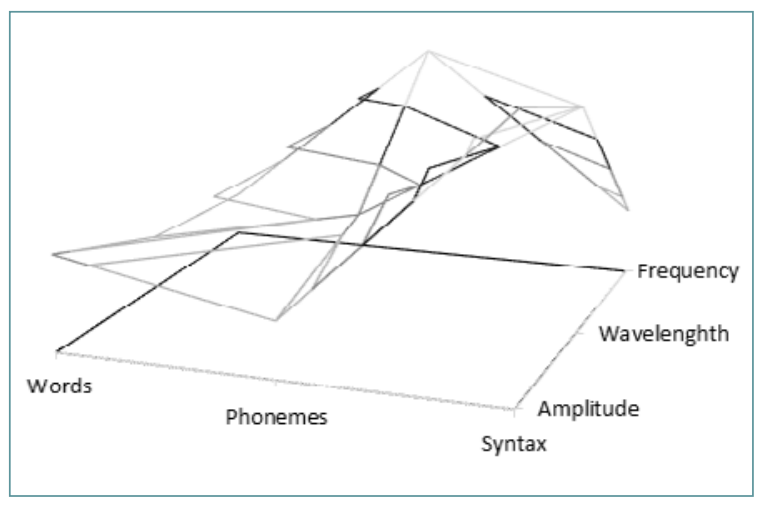

Figure 11: Pictorial representation of multidimensional dimensional language patterns.

Significantly, therefore, by mapping language in a multidimensional space, with time being a common factor, complex patterned signals emerge. These patterns are analogous to the patterns formed by odorant molecules in a chemical plume, where concentration can be analogously compared to acoustic features such as amplitude and tonality, movement of odour molecules is analogous to rhythm and timing, and each molecule in a chemical compound is analogous to a word or phoneme, with the relative composition of odour compounds being represented by syntax.

Furthermore, in nonhuman species, an anatomical structure which bears a remarkable similarity to the VNO is the 'melon', seen in melon-headed cetacean species, such as the beluga whale, Delphinapterus leucas. Interestingly, this structure is part of the nasal apparatus; however, its function is that of echolocation and receiving and transmitting acoustic signs (McKenna et al. 2012). A taxonomic group believed to have evolved from terrestrial even-toed ungulates, the Artiodactyla (Shimamura et al. 1997), cetaceans would have little use for an organ which processes atmospheric chemical signs in an aquatic environment. However, an adaptation of the VNO to detect acoustical signs would be an adaptive advantage in a marine environment, where sound is transmitted and reflected by environmental features such as the ocean bottom, various prey species, and conspecifics. Moreover, beluga whales, known as 'the canaries of the ocean', are famous for their complex and extensive vocal repertoire, including song 
(Garland et al. 2015). Thus, potential evidence for a unified hypothesis of sensory perception, where:

i) the sensory modalities are structurally inter-related and have evolved via divergent processes from one mechanoreceptor sensory system,

ii) acoustic communications are postulated to be 'frequency-mimicking' of odour and visual signs in the environment (Lewis 2020),

could be found in this aquatic mammalian species Indeed, a similar hypothesis has been put forward to explain the evolution of sensory processes in plants (Telewski 2006) and if occurring in animals, this hypothesis may be key in investigating language evolution.

However, it is noteworthy that because of the neurological integration of sensory modalities (Pennartz 2009), sensory unification at a mechanistic level (whilst a substantive hypothesis) is not necessary for the development of language from chemiosemiosis. Certainly, a potential framework for language evolution via complex pattern recognition, originating from chemiosemiosis and sensory integration, is already evident. For example, Stroop Colour and Word Tests, a psychological priming paradigm described by Bench et al. (1993), tests cross-modal sensory perception and word recognition. Further, Bench et al. (1993) were able to demonstrate that perception of a word as a sign is affected by factors such as the colour of the text. Similarly, an fMRI study conducted by González et al. (2006) revealed that in humans, referential words relating to odours elicited a response in the primary olfactory regions of the human brain. Moreover, a comprehensive review of multimodal food perception by Verhagen and Engelen (2006), reports some interesting findings. These include the results of a study by Zellner and Kautz (1990) on visual-olfactory integration, where subjects were found to be more likely to rate a solution as having a stronger odour if it was also coloured. Further Engen (1972) found that solutions without colour were more likely to be judged as odourless than solutions with the same odour, but which were coloured. Moreover, significantly, colour-odour integration has also been demonstrated by Kemp and Gilbert (1997), who found that subjects reported a solution to have a weaker odour if the hue of a previously viewed colour cue was of a low intensity.

Thus, these examples reveal that the remarkable extent of sensory integration (including olfaction) in humans, as well as previous examples in nonhuman species, can be demonstrated. Moreover, it is this sensory integration which allows for plasticity in sensory perception, and an integration of the olfactory apparatus, which is capable of highly complex multi-dimensional pattern recognition in both space and time, with the parts of the brain and nervous system responsible for communication and language.

\section{CONCLUSION}

Animal species across taxa are able to receive and process complex chemical signs and signals, for example, in the form of specific metal ligand functional groups, waxy esters, amino acids, and volatile organic compounds, with the information being transmitted simultaneously, to be 'caught' and analysed by the interpreter. In olfactory processes (which are closely linked to taste), this phenomenon is analogous to sending a written communication via email, allowing for data to be transmitted simultaneously, and then potentially stored in the environment to be detected by numerous interpreters, as in the case of urine scent marking. The complex physiological and cognitive perceptual sensory analysis of an odorant gives the chemical sign or signal a meaning, and, indeed, a relevance to the individual. It is this sense, which allows complex data from the environment to be detected and interpreted, in relation to foraging, predator/risk avoidance and gustation.

However, chemiosemiosis is not limited to detecting odours from the physical environment; as a form of social signalling, the process of chemiosemiosis can occur within or between individuals on a population or group scale. Chemiosemiosis is so fundamental that organisms actually manufacture and produce chemical signals to communicate between one another. Not only that, but some organisms are able to manipulate and exploit odours in the environment to use as signals. In summary, chemiosemiosis is fundamental to survival and reproduction, and is integrated with data from other sensory modalities to form another layer of multi-dimensional signal complexity.

Complex chemical signals, and their cognitive interpretation, are driven by pattern recognition of complex three-dimensional patterns that form in time as well as in space. Whilst in primitive single-cellular organisms this is most likely a stimulus-response process (similar to the attraction to sweet tastes, and repulsion from bitter tastes, observed in higher organisms and described by Peng et al. 2015), over evolutionary time, it is conceivable that in multicellular organisms, this would ultimately result in more complex pattern recognition. Further, when taking into account the multiple dimensions of language, including rhythm, prosody, syntax, and grammar, complex pattern recognition is the basis for highly developed cognition and language capabilities. Nonetheless, studies such as Rogers and Pullum (2011) tend to focus on linear sequences and string sets, and are centred around a vocal and aural evolutionary origin of language. However, when sensory modalities are integrated, and when language is analysed as a multidimensional phenomenon, complex three-dimensional patterns appear in both language and complex animal vocalizations. Furthermore, these vocal patterns can be seen to be analogous to the three-dimensional patterns produced in chemiosemiotic signs.

Olfactory sensory processes thus provide a potential basis for a hypothesis of cognitive, language, and speech evolution in which necessary cognitive and communicative capabilities originated as and developed from chemiosemiosis. It is conceivable that once the cognitive 
pre-requisites for processing complex signals were in place, providing the semiotic scaffolding for more complex and advanced semiotic transmissions, sensory integration led to vocal and visual forms of communication, which express those signals via other modalities. Moreover, a potential driving force for the evolution of such capabilities would likely be selection pressure on the signals themselves. For example, a gradual move from inhabiting a terrestrial environment surrounded by the atmosphere, to an aquatic environment where signals travel through water, as in the case of the Cetacea, gives rise to specific pressures on signals, and thus, on modes of signalling and communication.

As a hypothesis as to how language evolved, 'frequency-mimicking' (Lewis 2020) is a compelling idea, yet it is also possible that chance emotional vocalisations and behavioural signalling in response to emotive odorous signs (ZaldPardo 1997) could also have occurred; indeed, both could have occurred in tandem. Whatever the potential mechanisms, be it frequency-mimicking or emotional gestures and vocalisations, my proposed hypothesis, which relies on complex multi-dimensional signal processing, can concisely be termed a 'chemiosemiotic hypothesis of language evolution'. One of the initial ways forward to test such a hypothesis would be to isolate compounds in mixed odours, such as in the example of scent-rolling in domestic dogs and other species. Once common odour compounds have been isolated, and the odour composition analysed, then the meaning of individual scents can be extrapolated from behavioural studies, thus it may be possible to treat these odours linguistically. However, it is also likely that machine learning may hold the key to understanding these phenomena and testing the chemiosemiotic hypothesis of language evolution, as computer technology can handle the highly complex patterns associated with chemiosemiosis, and, indeed, language.

\section{REFERENCES}

Ackermann, H., Hage, S. R., Ziegler, W., 2014. Brain mechanisms of acoustic communication in humans and nonhuman primates: An evolutionary perspective. Behavioral and Brain Sciences, (6), 529-546.

Alexander, V. N., Grimes, V., 2017. Fluid biosemiotic mechanisms underlie subconscious habits. Biosemiotics, 10(3), 337-353.

Albuquerque, N., Guo, K., Wilkinson, A., Savalli, C., Otta, E., Mills, D., 2016. Dogs recognize dog \& human emotions. Biology letters, 12(1), 20150883.

Allman, B. L., Meredith, M. A., 2007. Multisensory processing in "unimodal" neurons: cross-modal subthreshold auditory effects in cat extrastriate visual cortex. Journal of neurophysiology, 98(1), 545-549.

Baird, B., 1986. Nonlinear dynamics of pattern formation \& pattern recognition in the rabbit olfactory bulb. Physica D: Nonlinear Phenomena, 22(1-3), 150-175.

Bench, C., Frith, C. D., Grasby, P. M., Friston, K. J., Paulesu, E., Frackowiak, R. S. J., Dolan, R. J., 1993. Investigations of the functional anatomy of attention using the Stroop test. Neuropsychologia, 31(9), 907-922.

Berwick, R. C., Okanoya, K., Beckers, G. J., Bolhuis, J. J., 2011. Songs to syntax: the linguistics of birdsong. Trends in cognitive sciences, 15(3), 113-121.

Boeckh, J., Ernst, K. D., 1987. Contribution of single unit analysis in insects to an understanding of olfactory function. Journal of Comparative Physiology, 161(4), 549-565.

Bonadonna, F., Sanz-Aguilar, A., 2012. Kin recognition and inbreeding avoidance in wild birds: the first evidence for individual kin-related odour recognition. Animal Behaviour, 84(3), 509-513.

Bonadonna, F., Miguel, E., Grosbois, V., Jouventin, P., Bessiere, J. M., 2007. Individual odor recognition in birds: an endogenous olfactory signature on petrels' feathers?. Journal of chemical ecology, 33(9), 1819-1829

Cafazzo, S., Natoli, E., Valsecchi, P., 2012. Scent-marking behaviour in a pack of free-ranging domestic dogs. Ethology, 118(10), 955-966.

Call, J., 2011. How artificial communication affects the communication and cognition of the great apes. Mind \& Language, 26(1), 1-20.

Caspers, B. A., Krause, E. T., 2011. Odour-based natal nest recognition in the zebra finch (Taeniopygia guttata), a colony-breeding songbird. Biology Letters, 7(2), 184-186.

Catala, A., Grandgeorge, M., Schaff, J. L., Cousillas, H., Hausberger, M. Cattet, J., 2019. Dogs demonstrate the existence of an epileptic seizure odour in humans. Scientific reports, 9(1), 1-7.

Chabrolles, L., Coureaud, G., Boyer, N., Mathevon, N., Beauchaud, M., 2017. Cross-sensory modulation in a future top predator, the young Nile crocodile. Royal Society open science, 4(6), 170386.

Chafe, W., Tannen, D., 1987. The relation between written and spoken language. Annual Review of Anthropology, 16(1), 383-407.

Clay, Z., Pople, S., Hood, B., Kita, S., 2014. Young children make their gestural communication systems more language-like: Segmentation and linearization of semantic elements in motion events. Psychological science, 25(8), 1518-1525.

Croll, R. P., 1983. Gastropod chemoreception. Biological Reviews, 58(2), 293-319.

Croset, V., Rytz, R., Cummins, S. F., Budd, A., Brawand, D., Kaessmann, H., Gibson, T. J., Benton, R., 2010. Ancient protostome origin of chemosensory ionotropic glutamate receptors and the evolution of insect taste and olfaction. PLoS Genet, 6(8), 001064.

Culbert, B. M., Gilmour, K. M., Balshine, S., 2019. Social buffering of stress in a group-living fish. Proceedings of the Royal Society B, 286(1910), 20191626.

Curtis, J. T., Liu, Y., Wang, Z., 2001. Lesions of the vomeronasal organ disrupt mating-induced pair bonding in female prairie voles (Microtus ochrogaster). Brain research, 901(1-2), 167-174. 
Dasgupta, S., Biswas, A., Abraham, A., Das, S., 2008. Adaptive computational chemotaxis in bacterial foraging algorithm. In 2008 International Conference on Complex, Intelligent and Software Intensive Systems (pp. 64-71). IEEE.

Derby, C. D., Kozma, M. T., Senatore, A., Schmidt, M., 2016 Molecular mechanisms of reception and perireception in crustacean chemoreception: a comparative review. Chemical Senses, 41(5), 381-398.

Døving, K. B., Trotier, D., 1998. Structure and function of the vomeronasal organ. Journal of Experimental Biology, 201(21), 2913-2925.

Emery, D. G., 1992. Fine structure of olfactory epithelia of gastropod molluscs. Microscopy research and technique, 22(4), 307-324.

Engen, T., 1972. The effect of expectation on judgments of odor. Acta Psychologica, 36(6), 450-458.

Favaro, L., Gamba, M., Cresta, E., Fumagalli, E., Bandoli, F., Pilenga, C., Isaja, V., Mathevon, N., Reby, D., 2020. Do penguins' vocal sequences conform to linguistic laws?. Biology Letters, 16(2), 20190589.

Gardiner, J. M., Atema, J., 2010. The function of bilateral odor arrival time differences in olfactory orientation of sharks. Current Biology, 20(13), 1187-1191.

Garland, E. C., Castellote, M., Berchok, C. L., 2015. Beluga whale (Delphinapterus leucas) vocalizations and call classification from the eastern Beaufort Sea population. The Journal of the Acoustical Society of America, 137(6), 3054-3067

Giuggioli, L., Bartumeus, F., 2010. Animal movement, search strategies and behavioural ecology: a cross-disciplinary way forward. Journal of Animal Ecology, 79(4), 906-909.

González, J., Barros-Loscertales, A., Pulvermüller, F., Meseguer, V., Sanjuán, A., Belloch, V., Ávila, C., 2006. Reading cinnamon activates olfactory brain regions. Neuroimage, 32(2), 906-912.

Gwinner, H., Berger, S., 2008. Starling males select green nest material by olfaction using experience-independent and experience-dependent cues. Animal Behaviour, 75(3), 971-976.

Havlíček, J., Roberts, S. C., 2013. The perfume-body odour complex: an insightful model for culture-gene coevolution?. In Dehnhard M. (Eds.), Chemical signals in vertebrates 12, New York, NY: Springer, pp. 185-195.

Horowitz, A., 2017. Smelling themselves: Dogs investigate their own odours longer when modified in an "olfactory mirror" test. Behavioural processes, 143, 17-24.

Hothersall, B., Harris, P., Sörtoft, L., Nicol, C. J., 2010. Discrimination between conspecific odour samples in the horse (Equus caballus). Applied animal behaviour science, 126(1-2), 37-44.

Jackson, D. E., Ratnieks, F. L., 2006. Communication in ants. Current biology, 16(15), R570-R574.

Jendrny, P., Schulz, C., Twele, F., Meller, S., von Köckritz-Blickwede, M., Osterhaus, A. D. M. E., Ebbers, J., Pilchová, V., Pink, I., Welte, T., Manns, M. P., 2020. Scent dog identification of samples from COVID-19 patients-a pilot study. BMC infectious diseases, 20(1), 1-7.

Jones, K. E., Dashfield, K., Downend, A. B., Otto, C. M., 2004. Search-and-rescue dogs: an overview for veterinarians. Journal of the American Veterinary Medical Association, 225(6), 854-860.

Kaissling, K. E., 1996. Peripheral mechanisms of pheromone reception in moths. Chemical Senses, 21(2), 257-268.

Keller-Costa, T., Canário, A. V., Hubbard, P. C., 2015. Chemical communication in cichlids: a mini-review. General and comparative endocrinology, 221, 64-74.

Kemp, S. E., Gilbert, A.N., 1997. Odor intensity and color lightness are correlated sensory dimensions. The American journal of psychology, 110(1), 35.

Lambrechts, M. M., Dos Santos, A., 2000. Aromatic herbs in Corsican blue tit nests: the 'Potpourri' hypothesis. Acta Oecologica, 21(3), 175-178.

Lewis, A., 2020. In Search of a Unified Theory of Sensory Perception: Possible Links between the Vibrational Mechanism of Olfaction and the Evolution of Language. Biosemiotics, 1-10.

Lisberg, A. E., Snowdon, C.T., 2009. The effects of sex, gonadectomy and status on investigation patterns of unfamiliar conspecific urine in domestic dogs, $\mathrm{Ca}$ nis familiaris. Animal Behaviour, 77(5), 1147-1154.

Lötsch, J., Kringel, D., Hummel, T., 2019. Machine learning in human olfactory research. Chemical Senses, 44(1), 11-22.

Malassis, R., Rey, A., Fagot, J., 2018. Non-adjacent dependencies processing in human and non-human primates. Cognitive Science, 42(5), 1677-1699.

Maraci, Ö., Engel, K., Caspers, B. A., 2018. Olfactory communication via microbiota: what is known in birds? Genes, 9(8), 387.

Maran, T., 2017. Mimicry and meaning: Structure and semiotics of biological mimicry, 16. Springer, Berlin.

McGuire, B., Bemis, K. E., 2017. Scent marking in shelter dogs: effects of body size. Applied Animal Behaviour Science, 186, 49-55.

McKenna, M. F., Cranford, T. W., Berta, A., Pyenson, N. D., 2012. Morphology of the odontocete melon and its implications for acoustic function. Marine Mammal Science, 28(4), 690-713.

Meredith, M. A., Stein, B. E., 1986. Visual, auditory, and somatosensory convergence on cells in superior coIliculus results in multisensory integration. Journal of neurophysiology, 56(3), 640-662.

Miller, L. R., Gutzke, W. H., 1999. The role of the vomeronasal organ of crotalines (Reptilia: Serpentes: Viperidae) in predator detection. Animal Behaviour, 58(1), 53-57.

Mollo, E., Fontana, A., Roussis, V., Polese, G., Amodeo, P., Ghiselin, M. T., 2014. Sensing marine biomolecules: smell, taste, and the evolutionary transition from aquatic to terrestrial life. Frontiers in chemistry, 2(92).

Nikonov, A. A., Butler, J. M., Field, K. E., Caprio, J., Maruska, K. P., 2017. Reproductive and metabolic state differences in olfactory responses to amino acids in 
a mouth brooding African cichlid fish. Journal of Experimental Biology, 220(16), 2980-2992.

Panda, H., 2010. Perfumes and Flavours Technology Handbook: Perfume making formulations, Delhi, India: Asia Pacific Business Press inc.

Peng, Y., Gillis-Smith, S., Jin, H., Tränkner, D., Ryba, N. J., Zuker, C. S., 2015. Sweet and bitter taste in the brain of awake behaving animals. Nature, 527(7579), 512-515.

Pennartz, C. M., 2009. Identification and integration of sensory modalities: neural basis and relation to consciousness. Consciousness and cognition, 18(3), 718-739.

Phillips, D. W., 1975. Distance chemoreception-triggered avoidance behavior of the limpets Acmaea (Collisella) limatula and Acmaea (Notoacmea) scutum to the predatory starfish Pisaster ochraceus. Journal of Experimental Zoology, 191(2), 199-209.

Pitcher, B. J., Briefer, E. F., Baciadonna, L., McElligott, A. G., 2017. Cross-modal recognition of familiar conspecifics in goats. Royal Society open science, 4(2), 160346.

Plenderleith, M., Oosterhout, C. V., Robinson, R. L., Turner, G.F., 2005. Female preference for conspecific males based on olfactory cues in a Lake Malawi cichlid fish. Biology Letters, 1(4), 411-414.

Proops, L., McComb, K., Reby, D., 2009. Cross-modal individual recognition in domestic horses (Equus caballus). Proceedings of the National Academy of Sciences, 106(3), 947-951.

Ortega, A., Zhulin, I. B., Krell, T., 2017. Sensory Repertoire of Bacterial Chemoreceptors, Microbiology and Molecular Biology Reviews Oct 2017, 81(4), e00033-17.

Queiroz, J., Emmeche, C., Kull, K., El-Hani, C., 2011. The biosemiotic approach in biology: Theoretical bases and applied models. Information and living systems: Philosophical and scientific perspectives, 91-130.

Ravignani, A., Dalla Bella, S., Falk, S., Kello, C. T., Noriega, F., Kotz, S. A., 2019. Rhythm in speech and animal vocalizations: a cross-species perspective. Annals of the New York Academy of Sciences, 1453(1), 79

Reed, R. R., 2004. After the holy grail: establishing a molecular basis for Mammalian olfaction. Cell, 116(2), 329-336.

Reiger, I., 1979. Scent rubbing in carnivores. Carnivore, 2(1), 17-25.

Richards, S., Goswami, U., 2019. Impaired recognition of metrical and syntactic boundaries in children with developmental language disorders. Brain sciences, 9(2), 33.

Rogers, J., Pullum, G. K., 2011. Aural pattern recognition experiments and the subregular hierarchy. Journal of Logic, Language and Information, 20(3), 329-342.

Sabiniewicz, A., Tarnowska, K., Świątek, R., Sorokowski, P., Laska, M., 2020. Olfactory-based interspecific recognition of human emotions: horses (Equus ferus caballus) can recognize fear and happiness body odour from humans (Homo sapiens). Applied Animal Behaviour Science, 230, 105072.
Saslow, C. A., 2002. Understanding the perceptual world of horses. Applied Animal Behaviour Science, 78(2-4), 209-224.

Schwenk, K., 1995. Of tongues and noses: chemoreception in lizards and snakes. Trends in ecology \& evolution, 10(1), 7-12.

Schmidt, R., Kunc, H. P., Amrhein, V., Naguib, M., 2008. Aggressive responses to broadband trills are related to subsequent pairing success in nightingales. Behavioral Ecology, 19(3), 635-641.

Shimamura, M., Yasue, H., Ohshima, K., Abe, H., Kato, H., Kishiro, T., Goto, M., Munechika, I., Okada, N., 1997. Molecular evidence from retroposons that whales form a clade within even-toed ungulates. Nature, 388(6643), 666-670.

Siljander, E., Gries, R., Khaskin, G., \& Gries, G. 2008. Identification of the airborne aggregation pheromone of the common bed bug, Cimex lectularius. Journal of chemical ecology, 34(6), 708-718.

Sorensen, P. W., Hara, T. J., Stacey, N. E., Goetz, F. W., 1988. F prostaglandins function as potent olfactory stimulants that comprise the postovulatory female sex pheromone in goldfish. Biology of Reproduction, 39(5), 1039-1050.

Sourakov, A., Duehl, A., Sourakov, A., 2012. Foraging behavior of the blue morpho and other tropical butterflies: the chemical and electrophysiological basis of olfactory preferences and the role of color. Psyche, 2012

Stevenson, R. J., 2010. An initial evaluation of the functions of human olfaction. Chemical senses, 35(1), 3-20.

Telewski, F. W., 2006. A unified hypothesis of mechanoperception in plants. American journal of botany, 93(10), 1466-1476.

Thünken, T., Waltschyk, N., Bakker, T. C., Kullmann, H., 2009. Olfactory self-recognition in a cichlid fish. Animal Cognition, 12(5), 717-724.

Tomás, G., Merino, S., Martínez-de la Puente, J., Moreno, J., Morales, J., Rivero-de Aguilar, J., 2013. Nest size and aromatic plants in the nest as sexually selected female traits in blue tits. Behavioral Ecology, 24(4), 926-934.

Tricas, T. C., Kajiura, S. M., Summers, A. P., 2009. Response of the hammerhead shark olfactory epithelium to amino acid stimuli. Journal of Comparative Physiology A, 195(10), 947-954.

lerusalimsky, V. N., Balaban, P. M., 2010. Two morphological sub-systems within the olfactory organs of a terrestrial snail, Brain Research, 1326, 68-74.

Verhagen, J. V., Engelen, L., 2006. The neurocognitive bases of human multimodal food perception: sensory integration. Neuroscience \& biobehavioral reviews, 30(5), 613-650.

Villamayor, P. R., Cifuentes, J. M., Fernandez de Troconiz, P., Sanchez-Quinteiro, P., 2018. Morphological and immunohistochemical study of the rabbit vomeronasal organ. Journal of anatomy, 233(6), 814-827. 
Vladimirov, N., Sourjik, V., 2009. Chemotaxis: how bacteria use memory. Biological chemistry, 390(11), 1097-1104.

Wang, L., Anderson, D. J., 2010. Identification of an aggression-promoting pheromone and its receptor neurons in Drosophila. Nature, 463(7278), 227-231.

Weeks, J. W., Crowell-Davis, S. L., Heusner, G., 2002. Preliminary study of the development of the Flehmen response in Equus caballus. Applied Animal Behaviour Science, 78(2-4), 329-335

Wells, D. L., 2012. Dogs as a diagnostic tool for ill health in humans. Alternative Therapies in Health \& Medicine, 18(2).

Wilson, D., Wharton, T., 2006. Relevance and prosody. Journal of pragmatics, 38(10), 1559-1579.

Wysocki, C. J., Preti, G., 2004. Facts, fallacies, fears, and frustrations with human pheromones. The Anatomical Record Part A: Discoveries in Molecular, Cellular, and Evolutionary Biology: An Official Publication of the American Association of Anatomists, 281(1), 1201-1211.

Yopak, K. E., Lisney, T. J., Collin, S. P., 2015. Not all sharks are "swimming noses": variation in olfactory bulb size in cartilaginous fishes. Brain Structure and Function, 220(2), 1127-1143.
Zald, D. H., Pardo, J. V., 1997. Emotion, olfaction, and the human amygdala: amygdala activation during aversive olfactory stimulation. Proceedings of the National Academy of Sciences, 94(8), 4119-4124.

Zelano, C., Sobel, N., 2005. Humans as an animal model for systems-level organization of olfaction. Neuron, 48(3), 431-454.

Zellner, D. A., Kautz, M. A., 1990. Color affects perceived odor intensity. Journal of Experimental Psychology: Human Perception and Performance, 16(2), 391.

Zeng, Y., Lou, S. L., Liao, W. B., Jehle, R., Kotrschal, A., 2016. Sexual selection impacts brain anatomy in frogs and toads. Ecology and Evolution, 6(19), 7070-7079. Websites

Gray, R. 2017. The Many Reasons Why Dogs Might Roll in Smelly Poo, BBC. Available at: <http://www.bbc. com/earth/story/20170608-the-many-reasons-why-dogs-might-roll-in-smelly-poos.

Return to front page $\uparrow$ 